\title{
Supercomputers Keeping People Warm in the Winter
}

\author{
Mario Romero \\ Dept. High-Performance Computing \\ and Visualization \\ KTH Royal Institute of Technology \\ Stockholm, Sweden \\ marior@kth.se
}

\author{
Hanna Hasselqvist \\ Dept. Media Technology and \\ Interaction Design \\ KTH Royal Institute of Technology \\ Stockholm, Sweden \\ hannaha@kth.se
}

\author{
Gert Svensson \\ PDC Center for High-Performance \\ Computing \\ KTH Royal Institute of Technology \\ Stockholm, Sweden \\ gert@pdc.kth.se
}

\begin{abstract}
We present the design and evaluation of the heat recovery system for KTH's Lindgren, Stockholm's fastest supercomputer, a Cray XE6. Lindgren came into service in 2010 and has since been primarily used for complex numeric simulations of fluid mechanics and computational chemistry and biology. The heat exchange system collects the wasted heat from Lindgren's 36,384 CPU cores and transfers it via the standard district heating and cooling system to a neighboring building which houses the Chemistry laboratories. We analyze the impact of Lindgren's heat recycle system as a function of outside temperature and we estimate the system's carbon emission savings. Since the original installation of Lindgren in 2010, it has become common practice to use water cooling systems for supercomputers, as water is a better heat transfer medium than air. We discuss the relevant design lessons from Lindgren as they relate to practical and sustainable waste heat recovery designs for today's platforms. Finally, we estimate that the recovered heat from Lindgren reduced the carbon emissions by nearly 50 tons over the 2012-13 winter, the sample period of our analysis.

Index Terms - Supercomputer, data center, waste heat recovery, heat exchanger, waste energy reuse, urban district heating and cooling, high-performance computing.
\end{abstract}

\section{INTRODUCTION}

Supercomputers and data centers consume enormous amounts of electrical power. Worldwide, these centers currently absorb roughly 30 billion watts of electricity, or $1.3 \%$ of the energy demand of the world. This is enough to provide the average electricity needs of over 60 million world citizens $[1,2]$. Unfortunately, most of this energy becomes heat that is typically wasted. According to the November 2013 Top500 report, the fastest supercomputer in the world as of this writing is the Chinese Tianhe-2 (Milkyway-2) [3]. Even after extensive work in improving its power efficiency, Tianhe-2 consumes 17.8 MW of electricity. This one computer consumes enough energy to provide the average electricity demands of over 47,000 people in China [2].

Heat disposal, in fact, is a paramount concern in the design and deployment of supercomputer centers. Typically, industrial ventilation systems are erected to dissipate the heat away from the servers, in practice simply wasting it into the atmosphere. Moreover, these refrigeration systems consume up to $50 \%$ of the energy used in the center as a whole. Historically, the single most important goal for supercomputers has been improving their speed, conventionally measured in floating point operations per second (FLOPS). Tianhe-2 soars twice over its runner up at 33.8 Peta-FLOPS, which is 30 times the performance of the fastest supercomputer five years back and 270,000 times the performance of the fastest supercomputer 20 years back [3]. This drive has created the ever increasing power-hunger and heat-waste culture surrounding supercomputers.

Only as recent as 2008 did the issue of waste heat recovery start taking center stage [4]. At the time, the primary refrigeration technology deployed ventilation systems, using air as the heat sinking medium. In 2009, the engineering design team of Lindgren tailored an air-to-water transfer system that today evacuates the heat from the supercomputer and injects it into the existing district heating system, targeting an adjacent building at the campus, the Chemistry Laboratory. Key design considerations included the Nordic winter, the urban setting with district water heating and cooling systems, the short distance between the buildings, and the fact that due to air quality safety standards, the Chemistry Laboratory requires higher-than-usual fresh-air ventilation. This ventilation requirement raises the energy demands of the air heating system of the building and, by design, uses more of the recycled heat from Lindgren.

The contributions of this paper are:

The design rationale behind Lindgren's heat transfer system in the context of sustainability in a Nordic academic urban environment;

- An evaluation of the environmental impact of the deployment of Lindgren's heat recovery system over the cold 158-day period during the 2012-13 Stockholm winter;

- A discussion on the design lessons for the sustainable future of supercomputing.

In section II, we contextualize Lindgren's heat recovery system within the state-of-the-art of 2009 - 2014 and we review the underlying engineering values of the community of supercomputer heat management. In section III, we describe the design of the Lindgren heat recovery system and focus on its sustainability rationale, theory, and pragmatics. In section IV, we present the evaluation of the environmental impact of the deployment of Lindgren's heat recovery system. In section $\mathrm{V}$, we present a discussion of the design lessons of the installation and operation of the heat recovery system. We conclude and point to future work in section VI. 


\section{RELATED WORK}

While the top500 ranking of the fastest supercomputers has existed since 1993, the green500 ranking of the most efficient supercomputers has only existed since 2007 [3, 5]. This statistic alone heralds the shifting of current priorities. The green500 focuses on metrics of efficient performance. While the community of supercomputer experts has yet to agree on a single metric of efficiency, the most commonly used measure is the number of floating point operations per second per watt $[6,7,8]$. Other metrics considered include the Total Cost of Ownership (TCO), which aggregates factors such as energy, space, reliability, and availability, and Power Usage Effectiveness (PUE), defined as the total facility energy for the data center divided by the energy used by the IT equipment.

Figure 1 plots efficiency vs. performance by year. The general trend in computing is increasing efficiency, evidenced by the upward push over the years. A few supercomputers push disproportionately for performance. They are the points below the green diagonal in the graph, including Tiannhe-2, the upper-right black dot. The diagonal in the graph is the practical boundary of power and scale limitations [7]. It is notable that the November 2013 edition of the Green500 list was the first where the average power used by the supercomputers on the list decreased compared to the previous edition [5].

While the general trend in computing both at the pocket and at the supercomputing scale pushes for efficiency, the reality of large-scale computing is that energy consumption and heat management are an increasing paramount concern. The estimated annual increase in demand on power consumption is 15-20\% [9]. Furthermore, highly-integrated computing devices will increase power consumption spatial density from 8 to 15 $\mathrm{kW} / \mathrm{m}^{2}$ [10]. According to Brunschwiler et al., two important components in moving towards zero-emission supercomputer centers are more efficient cooling and reuse of the centers' waste heat [9]. The central theme of this paper focuses on recovering wasted heat to increase the net efficiency of the system, push for sustainability by recycling wasted heat, and reduce the cooling costs which typically account for up to $50 \%$ of the energy consumption costs. The most current and comprehensive review of heat recovery supercomputing and data center systems is Ebrahimi et al. [4].

Large-scale computing centers primarily employ three types of cooling systems: 1) air-cooled; 2) water cooled; and 3) two-phase cooled systems. The centers that recover the wasted heat employ a number of methods for recycling the heat, primarily: 1) plant or district heating / hot water production; 2) power plant co-location; 3) absorption cooling; 4) organic rankine cycle; 5) piezoelectrics; 6) thermoelectrics; 7) desalination / clean water production; and 8) biomass colocation. Here, we focus on hot water production for district heating from air-cooled systems.

In this paper, we present a hybrid cooling design that uses air cooling as a first step and air-water heat exchange in a second step. Typically, air-cooled systems, the majority of computer centers today, have a cold and a hot aisle. Cold air is pushed from the cold aisle through the rack into the hot aisle, where it is collected and driven back to the computer room air

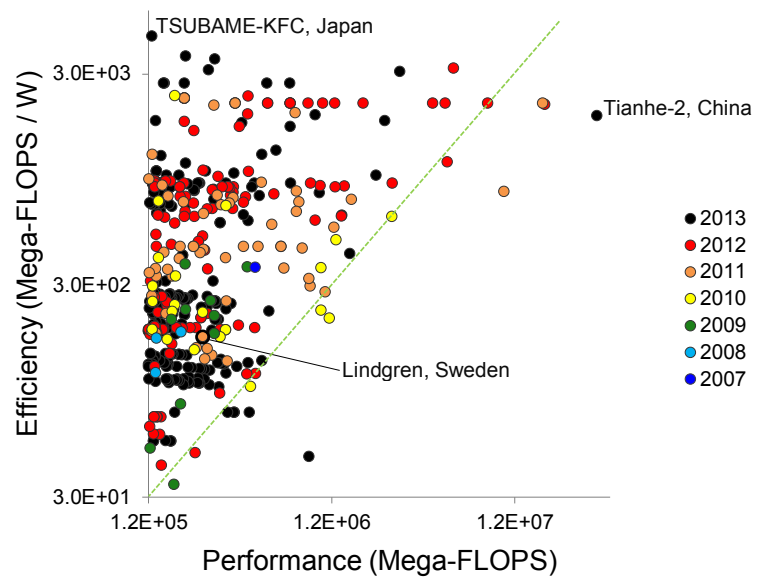

Fig. 1. Green500 November 2013 efficiency vs. performance by year of deployment [5]. The global trend pushes growth vertically. A few machines below the green diagonal push primarily for performance, including Tianhe-2, the upper-right point.

conditioning units (CRACs). Some centers push the heat from below through raised floors and collect the hot air through convection. Lindgren uses this system, except it collects the warm air into heat exchangers to recover and recycle the heat. Khalifa et al. present a recent energy optimization techniques for air-cooled data centers [11]. The paper presents a simplified thermodynamic model that identifies optimal, energy-efficient designs and operating scenarios and parameters such as flow rates and air supply temperatures.

Liu et al. coined the term "data furnace" to refer to computer centers that heat nearby buildings or homes [12]. In this respect, Lindgren is the Chemistry building's data furnace. Liu effectively demonstrates that data furnaces exhibit three advantages over traditional computer centers: 1) a smaller carbon footprint; 2) reduced total cost of ownership per server; and 3) closer proximity to the users. In this paper, we demonstrate these advantages plus a forth one: increased reliability, as the recycling system supports center cooling even when the district cooling systems has stalled.

There exist a number of practical data furnaces, especially in Scandinavia. For example, Helsinki's Academica has set up a server that provides heating to 500 homes [13]. Stockholm's Interxicon data center uses seawater for cooling and is reportedly saving $\$ 1 \mathrm{M} /$ year [14]. Recently, Dutch startup Nerdalize proposed "to replace home radiators with disguised computer servers and sell data processing time to remote users while the excess heat warms the house for free" [15].

Ebrahimi argues that the integration of a computer center to district heating requires tailored design and analysis. Our experience supports this claim. Furthermore, the authors summarize the following issues: 1) reusing waste heat requires minimum supply temperatures from as low as $35{ }^{\circ} \mathrm{C}$ when directly heating air to $70{ }^{\circ} \mathrm{C}$ when directly applying to hot water supply; 2) benefits of heat reuse for air and water heating include a reduction on operating costs and potential income from selling the heat; and 3) the challenges include greater system complexity and possible maintenance and security issues. Finally, district heating may not be available, depending on the location of the center. 


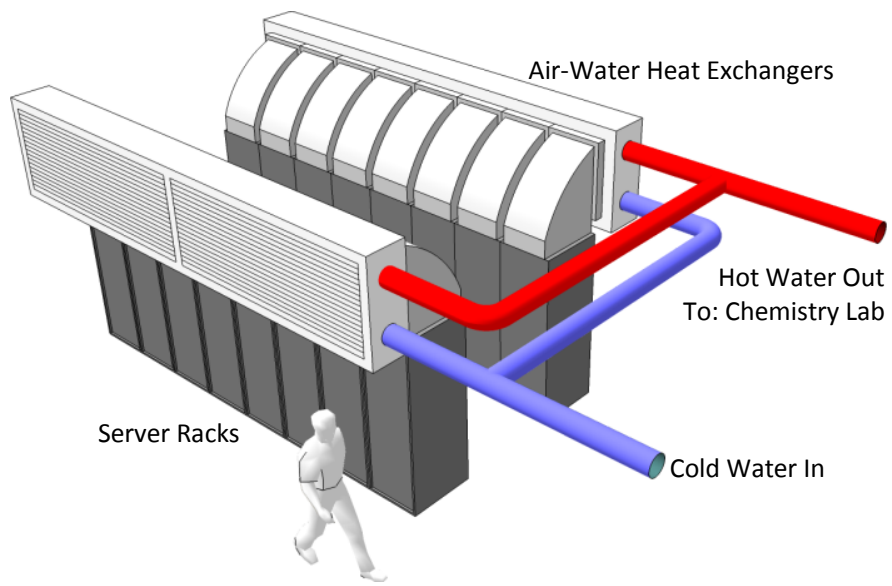

Fig. 2. PDC, Center for High-Performance Computing, KTH. Lindgren is a CRAY XE6 composed of 16 racks. Lindgren's heat recovery system is based on an industrial air-water heat exchanger that sends the exhaust heat to the neighboring Chemistry Laboratory building.

\section{DESIGN OF LINDGREN'S HEAT RECOVERY SYSTEM}

Figures 2-4 show the final design of Lindgren's heat recovery system. Cold water from district cooling enters the computer center and feeds into the computer room air conditioners (CRACs) and into Lindgren's industrial air-water heat exchangers (in the case that the returning water from the Chemistry building is not sufficiently cooled). The CRACs pump cold air $\left(16^{\circ} \mathrm{C}\right)$ through the raised floor underneath the supercomputer racks. The air travels up through the racks collecting the heat generated by the servers. The hot air $(35-$ $44{ }^{\circ} \mathrm{C}$ ) exiting the racks enters the large ducts connected to the heat exchangers sitting on top of the racks. In the heat exchangers, incoming water $\left(16^{\circ} \mathrm{C}\right)$ receives the heat from the hot air traveling up and out. The exchanger outputs air at roomneutral temperature back into the room $\left(21{ }^{\circ} \mathrm{C}\right)$, which is cooled again by the CRACs and the cycle continues. The heat exchanger also outputs warm water $\left(30-35^{\circ} \mathrm{C}\right)$ that is transported to the Chemistry Laboratory building for heating.

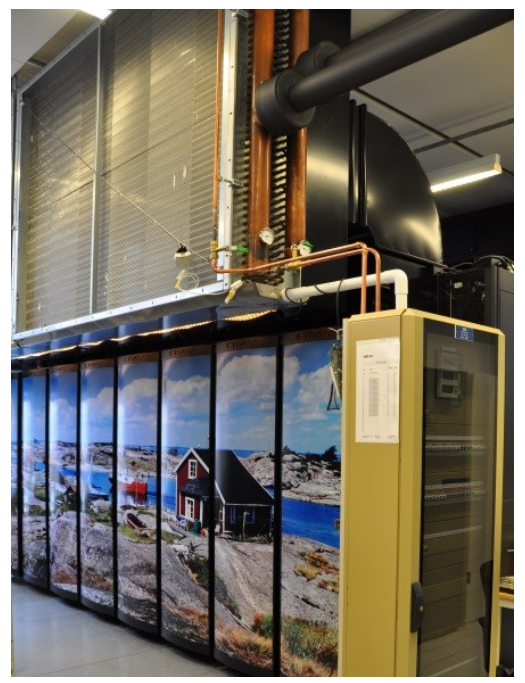

Fig. 3. Lindgren's industrial air-water heat exchanger-based energy recovery system.

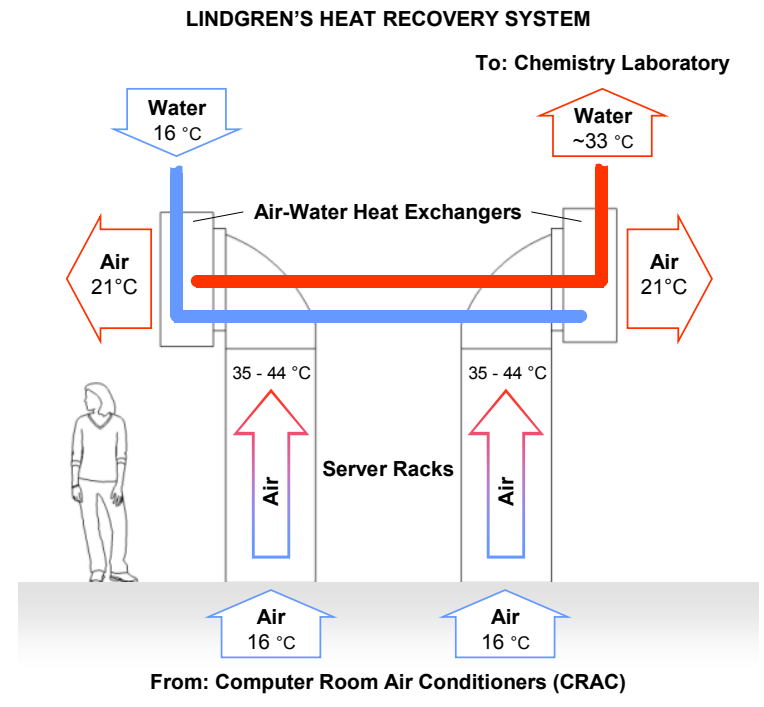

Fig. 4. Lindgren's heat recovery system sends recycled heat to Chemistry building through industrial air-water heat exchangers.

Next, we summarize the background, the design challenges, and the solution rationale previously detailed in [16].

\section{A. Background}

PDC Center for High-Performance Computing is the major of six academic supercomputer centers in Sweden. It's hosted by KTH Royal Institute of Technology in Stockholm. Before the installation of Lindgren, a 16-cabinet Cray XE6, in 2010, PDC consumed $800 \mathrm{~kW}$ of electric power. Lindgren increased consumption to roughly $1300 \mathrm{~kW}$.

Figure 5 shows the historical and projected increase in energy demand at PDC. This ever-increasing energy consumption is a common and central challenge to all supercomputer and data centers. In 2009, PDC consumed an estimate of $7.0 \mathrm{GWh}$. In Stockholm, electric power is available for roughly $0.1 € / \mathrm{kWh}$ [17]. At the current consumption rate of $11.4 \mathrm{GWh} /$ year, PDC spends roughly 1.14 million $€ /$ year and this is projected to grow to 1.8 million $€ /$ year by 2016 .

Cooling is another significant design challenge of large-

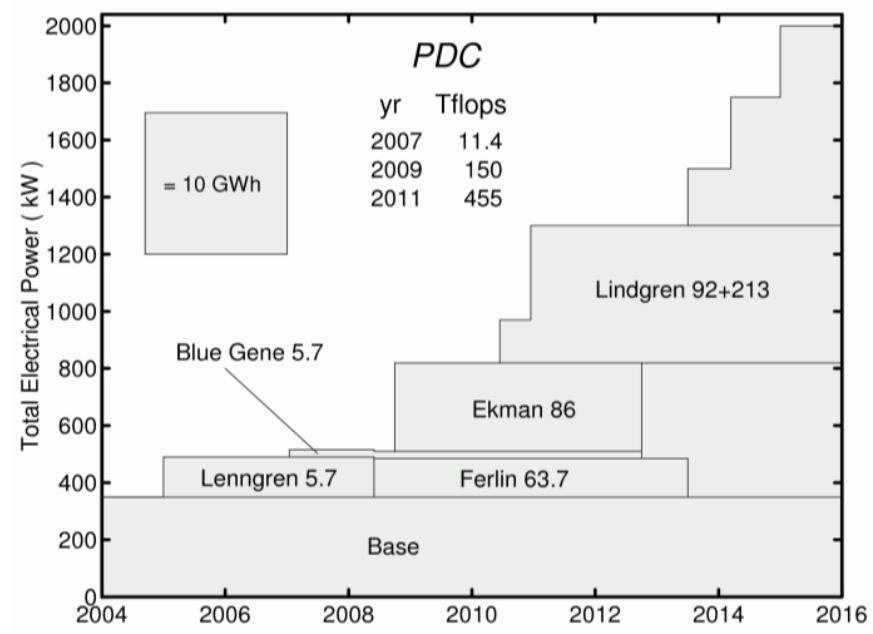

Fig. 5. Historical and projected energy demand growth at PDC. 
scale computing centers. Up to $50 \%$ of the energy consumed in a center goes to cooling systems. The heat disposal of PDC's computer hall when it is not used to heat the Chemistry building comes from district cooling available in Stockholm. The input temperature from the district cooling is $8{ }^{\circ} \mathrm{C}$ to the computer room. Outgoing water temperature must be at least $10{ }^{\circ} \mathrm{C}$ higher according to the regulations of the energy company. This is already an environmentally friendly method of cooling, since the company distributing the cooling water produces district heating with heat pumps from the returning (warmer) water. Additional cooling is done by seawater. However, KTH not only does not get reimbursed for produced heat, it has to pay $0.05 € / \mathrm{kWh}$ to get rid of the energy. Moreover, during the cold season in Stockholm, KTH has to pay for district heating from the same energy company at 0.07 $€ / \mathrm{kWh}$.

Since the distinct cooling is less reliable than what is required for computer operation, the center maintains a backup method of emergency cooling without using district cooling. Locally produced cooling is limited mainly by space limitations. Many $\mathrm{KTH}$ campus buildings are protected as historical landmarks, including PDC's building. Significant modifications, including large outdoor equipment, are partially or entirely forbidden. PDC uses tap water directly or with compressor cooling as emergency cooling.

In 2009, the computer room used two methods of cooling: 1) low-density equipment was cooled by ambient cooling from ordinary Computer Room Air Conditioners (CRAC) units; and 2) high-density equipment was cooled by hot-aisle encapsulated cooling from APC [18].

Our initial design goal was to increase present and future system sustainability and affordability by improving the heat transfer efficiency and by recovering the heat through a recycling mechanism. We aimed at minimizing our dependency on district heating and cooling. The 2010 cooling cost was around $45 \%$ of the electricity expenditure at PDC.

\section{B. Design Challenges}

We had to solve five main challenges: 1) finding a supercomputing system with practical operating exhaust temperature ranges; 2) capturing the supercomputer's outgoing heat with minimal waste; 3) sharing the physical space between the supercomputer and the industrial heat exchange system; 4) finding a practical and efficient transfer system to the site of the consumer; and 5) finding a valuable energy consumer that could reuse the heat.

\section{1) Finding the right supercomputer}

The 2009 methods of cooling presented in the related work section produced a water temperature of $18^{\circ} \mathrm{C}$, too low to heat normal buildings. It occurred to us that we could use heat pumps to increase the temperatures, yet this would have involved further use of electricity. Our calculations showed this to be barely economical.

On the other hand, the Cray XE6 air-cooled supercomputer system we investigated took in cold air $\left(16^{\circ} \mathrm{C}\right)$ from under the raised floor and exhausted it to the top of the racks with a temperature range of $35-45{ }^{\circ} \mathrm{C}$ under normal operation conditions. We had found a system with practical exhaust temperature ranges in the Cray XE6. The next challenge became capturing this heat.

\section{2) Capturing the exhaust heat}

The challenge was to capture the exhaust heat without losing too much temperature. We investigated industrial airwater heat exchangers and calculated that they would produce useful outgoing water temperatures. The industrial exchangers we needed were large, so the next challenge was placing them in the same room, with severe space limitations.

\section{3) Sharing the physical space}

Efficient exchangers tend to be large and heavy and contain water with its ever-present leakage potential - a very nefarious menace to any computer, in general, and to mega-charged supercomputers, in particular. We investigated a number of options. First, we considered placing the exchange units beside the Cray on the same row. Next, we discussed placing the exchange units between the racks. Yet, all the options sharing the floor space required considerable computing center footprint, beyond what we could afford. Furthermore, placing the exchangers between the racks meant Cray would have to change the cable layout of the system, an unviable option.

We considered placing the exchange system directly above the computers to be too risky due to potential leakages. After considerable argumentation, we decided to face the challenge of designing a raised heat exchange system. It was large, heavy, and full of water and it would hang over the principal scientific computing asset of Scandinavia.

The final solution was to hang the heat exchangers from the ceiling not directly on top of the Cray racks, but slightly displaced so that they do not vertically overlap (see figures 24). This design does not fully eliminate the threat of water leaking into the racks, but it reduces the consequences of small leaks and provides greater access for regular inspection.

Hot air from the top of the Cray feeds into the heat exchangers via chimney-like ducts. We checked the throughput of the Cray system fans and found them to be strong enough. Coincidentally, the design of the fans incorporated the need for the extra pressure drop of the cooling coils in Cray's liquid cooling solution.

Due to the size of the room, we decided to place the sixteen Cray racks in two rows. The resulting eight racks were too large for a single heat exchanger. Thus, we distributed four heat exchangers over the sixteen Cray racks in groups of two (see figures 2 and 3 ).

\section{4) Finding a practical heat transfer system}

We decided to use the district heating and cooling systems to transfer the heat to a neighboring building. Economically, this option presented a practical solution. Most of the buildings in Stockholm share the district heating system. Using the district system was the natural choice for heat transfer, yet it needs to be a consideration in places without district systems.

Nevertheless, we could not pump the outgoing water at 33 ${ }^{\circ} \mathrm{C}$ into the hot water lines of the district heating at $60{ }^{\circ} \mathrm{C}$ as that would have actually been detrimental to heating. We came to a practical solution by pumping the warm water into the cooling lines. This only works in the winter. We close the cold water loop from district cooling and reverse the flow of heat from PDC to the destination building (see figures 6 and 7). The 


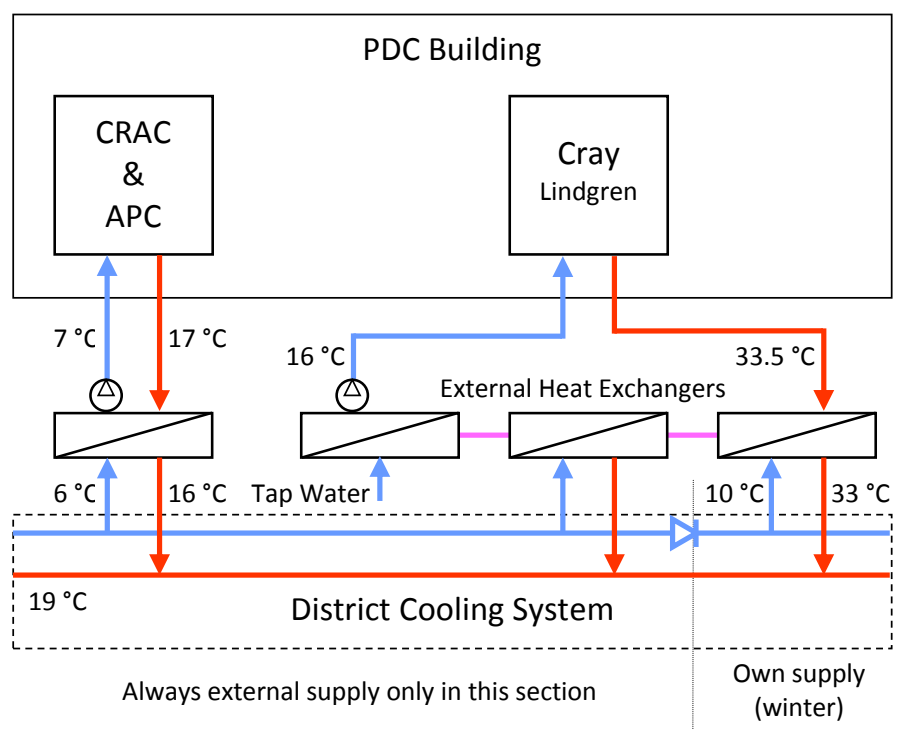

Fig. 6. Layout of water cooling in PDC: Ambient cooling via CRAC-units (left) and hot aisle APC connected to district cooling (with back-up). Also seen are the external heat exchangers serving the Cray racks with high temperature cooling downstream the check valve. System backed up by conventional district cooling and tap water cooling.

next challenge was finding the best candidate for receiving the heat from PDC.

5) Finding a valuable destination for the heat

After considerable search, we struck gold with the Chemistry Laboratory building at KTH. First, the chemistry building is physically near the PDC building, thus making heat transfer between the two buildings a feasible task. Second, both buildings share the same ring of the district cooling pipes, making the transfer medium an existing and practical solution. We did not have to build a new transfer medium. Third, the Chemistry building has the special requirement of removing potentially hazardous fumes through extra aeration. This translates to higher energy demands to heat the incoming outdoor fresh air during the winter as the building needs more fresh air than its counterparts on campus. Finally, the chemistry building was undergoing renovations, which allowed us to tap into its exposed systems without extra costs.

\section{Considerations of the Final Design}

Since its deployment and calibration, the heat recovery system has worked remarkably well. In four years, we have not had a single interruption caused by the cooling system. In fact, during instances when the district cooling has failed and the outdoor temperature has been low enough, we have been able to cool the Cray with the heat reuse system. When reusing heat, the cooling system is designed for a forward temperature of $20{ }^{\circ} \mathrm{C}$. Furthermore, the normal forward temperature of the district cooling system is $8{ }^{\circ} \mathrm{C}$. Thus, this temperature can vary substantially before triggering the emergency cooling. These features make the cooling system highly stable and reliable.

In the following subsections, we present the main experiences and lessons of operating with the final design of the heat recovery system.

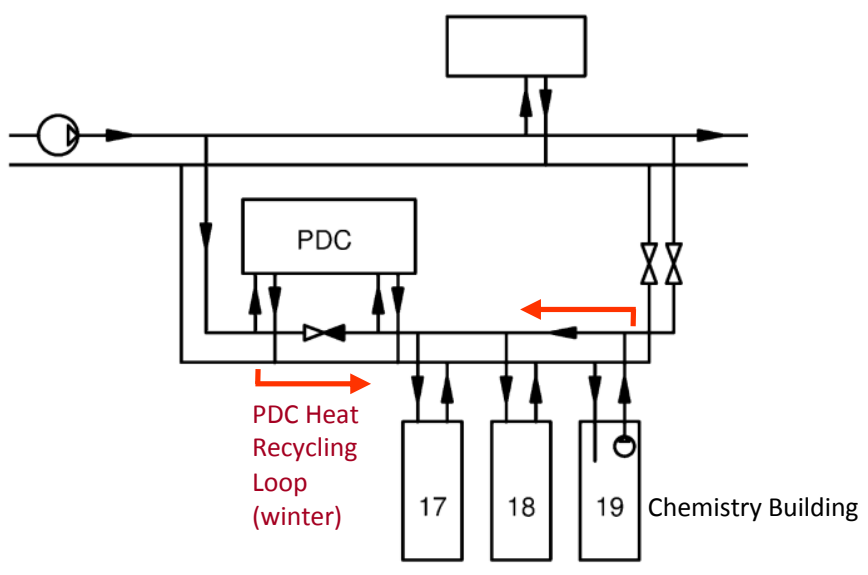

Fig. 7. Ring-shaped cooling system at the KTH campus. As the check valve closes, a self-supplying section emerges between PDC and buildings 17, 18, and 19. The Chemistry Laboratory, building 19, is capable of taking care of all recycled heat in the section at outdoor temperatures below $0{ }^{\circ} \mathrm{C}$. PDC uses coolant from both sides of the check valve.

\section{1) Cooling the water for the heat exchange system}

The Cray exhausts heated air through the outlets at the top of the racks at $35-45{ }^{\circ} \mathrm{C}$. The heated air travels via the ducts to the heat exchangers. In the heat exchangers, the air is cooled to around $21^{\circ} \mathrm{C}$ and exits into the room. The transfer heats the water from around $16^{\circ} \mathrm{C}$ to $30-35^{\circ} \mathrm{C}$. The water circulates in a closed loop that can be cooled by three different heat exchangers placed outside the computer room: a first exchanger for heat reuse; a second exchanger for district cooling; and a third exchanger for ordinary tap water if everything else fails (see figure 6). In general, the heat re-use system is controlled by the building control system. However, the emergency cooling and the valves in the computer room are controlled by a stand-alone regulator to remain reliable through completely independence from other parts of the system.

\section{2) Rethinking control systems}

Another problem was to understand the overall control of the system, which is distributed over many independent control systems. One typical example was the previous control of the under-floor temperature in the computer room. This was expected to be handled by the old CRAC units. If they get an intake air temperature of $22^{\circ} \mathrm{C}$ they produce just below $16^{\circ} \mathrm{C}$, which is acceptable. However, the measurement happens only on the intake. This measure also controls the start of the emergency cooling in the CRAC units. This means that when a CRAC unit fails, the under-floor temperature increases, and the outgoing temperature from the heat exchangers above the Cray increases. This forces the control system for the heat exchangers to increase the opening of the valves on the water. The temperature of the room is brought back to $22{ }^{\circ} \mathrm{C}$. Everything seems normal and the emergency cooling is never started. However, the under-floor temperature is now too high, and the Cray can get damaged or shut down. Thus, the control of the CRAC units had to be changed. This was done by physically moving the temperature sensors to under the floor. In the first attempt the sensors were placed just under the Cray. This didn't work so well because a sensor belonging to one CRAC unit was affected too much by the other units. 
Therefore, the working solution was to pace the sensors close to each CRAC unit, but still under the floor.

\section{3) Connecting to the Chemistry building}

Laying down new pipes would be expensive. The idea to use existing distribution systems to transfer recycled heat seems natural in hindsight. The campus receives and distributes district heating and cooling water through a single point. There exist operating limits to feeding water to these systems. The water feeding the heating cycle must be warmer than $60^{\circ} \mathrm{C}$ and the water feeding the cooling cycle must be colder than $10^{\circ} \mathrm{C}$. The critical design affordance is that there is a single requirement for the return lines: the temperature of the cooling water must be raised by at least $10{ }^{\circ} \mathrm{C}$ in the district cooling circuit. By operating within these requirements, it was possible to use the existing pipes in the cooling system.

Among conventional waste heat sources such as watercooled low-temperature chillers, air- and helium-condensing plants, distillers, compressors and testing equipment in physics and material science, the supercomputer center represents by far the largest single cooling water user on campus. Of a total of 2,000 kW, PDC uses 1,300 kW. This high-cooling load requires large amounts of circulating water.

The key design insight was using the return pipe of the water cooling system as a source of low-grade heat. To foster reliability and capacity, the cooling grid on campus is a looping network. Fortunately, this looping design also affords the sustainability goal of recycling low-grade heat.

The computer center and the Chemistry building are on the same loop. Through key valves, we close a section of this loop, and place the computer center in the upstream direction. We placed a check valve in the feed line outside the computer center as shown in figures 5 and 6 .

During the summer, the check valve is open and all cooling demands are supplied from the city. When operating the system for heat reuse, the flow direction in the intermediate section of the network reverses. The recovered heat enters the return line of the district cooling system and flows towards the Chemistry building, literally reversing the designed flow of the cooling lines. At outdoor temperatures below $0{ }^{\circ} \mathrm{C}$, all recovered heat is recycled and the check valve closes. The section between the valves (red loop in figure 7) forms an isolated system solely transfering the waste heat, now recycled and with a reversed flow direction. At warmer weather, the check valve remains open, leaving the flow direction to be only partially reversed.

The outgoing return temperature from the computer center is roughly $30-32{ }^{\circ} \mathrm{C}$. Unfortunately, the path to the Chemistry building involves two intermediate cooling loads (buildings 17 and 18 in figure 7) with significantly lower return temperatures. The temperature of the water arriving to the Chemistry building is roughly $27{ }^{\circ} \mathrm{C}$. This effect is more pronounced at higher outdoor temperatures, when it is possible to reuse only a minor fraction of the Cray computer heat. The recovered heat arriving at the Chemistry building may drop to $20-23{ }^{\circ} \mathrm{C}$. Yet, this is still within operating limits. The Chemistry building's circulating air must be heated to $18{ }^{\circ} \mathrm{C}$ and the required power during these conditions is relatively low.

\section{4) Using the recycled heat in the Chemistry building}

From the idea's inception, we regarded the Chemistry building as a suitable recipient of heat from PDC. Like most buildings of this kind in Stockholm, the edifice possesses a heat recovering ventilation system. However, in this case, the heat recovery suffers from the mismatch in flow caused by the widespread use of separate process ventilation without any heat re-use in the building. The inlet air requires extra heating due to the difference in flow rates of the opposing flows in each air handling unit. Fortunately, the temperature requirements on this additional heat are low. A heat source with a temperature as low as $15-25^{\circ} \mathrm{C}$ is sufficient.

The heat is supplied via a secondary system heated by water from the warm return line of the cooling system. Whenever the temperature in this secondary system drops below $10{ }^{\circ} \mathrm{C}$, it becomes possible to maintain the required temperature allowing the water to be pumped back in the feed line of the cooling network.

\section{Design Lessons}

1) Use existing infrastructure to mediate sustainability, even if it means getting creative

Our equally important goals were reducing operation costs and increasing the environmental sustainability of our future supercomputing systems. While we could have constructed infrastructure to secure these goals in the long run, we chose to exploit, as much as possible, the existing infrastructure, thus limiting the implementation cost and the environmental impact of construction. Rather, we needed to exploit existing affordances, pipes and valves, and produce the counterintuitive solution of reversing the flow of water in the cooling system during the winter and turning a subsection of the cooling infrastructure into a low-grade heat recycling system.

2) Encapsulate module interfaces to account for different scales between interconnected systems

The designed heat recovery system is complex. We made many choices with minimal previous experience to refer to. The coauthors of this paper are computer scientists. A collaboration challenge with cooling and energy experts is that they typically work on buildings that last hundreds of years. They design installations that are functional for 30 years or more. On the other hand, the lifecycle of a supercomputer is only four years. After this period, it becomes obsolete. Thus, we will soon (2014) replace the supercomputer and most of the parts directly attached to it. The design of the two systems needs a stable interface that affords modular development, especially on the computer's side, similar to the encapsulation and plug-and-play concepts from computing.

3) Communicate early, more often, and in greater detail with the equipment vendors

An additional problem was that computer vendors normally do not produce custom installations. It may be hard to get the correct and up-to-date information about details that ordinary customers do not require. In our case, we had to have exact measurements to fit the hood onto the Cray rack. We visited the vendor in Finland exclusively to take measurements and ascertain that everything would fit. Cray was also very helpful 


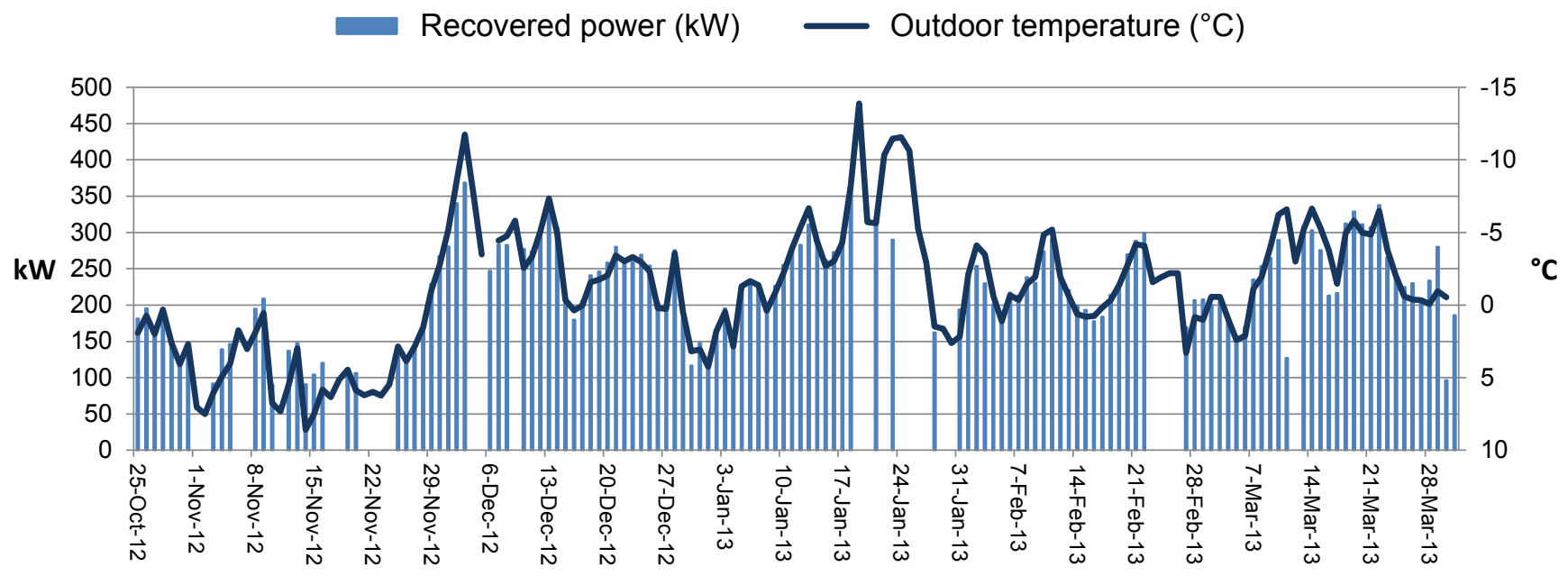

Fig. 8. Comparison of recovered power and outdoor temperature. Please note that the temperature scale is inverted. Empty columns are missing data.

in providing information and drawings. Nevertheless, they missed informing us about a minor mechanical design change on the top of the rack. Fortunately, it was possible to make the required changes of the ducts on site. Synthesizing, designers need to communicate early and closely with the computer vendor, stressing the required tailoring of the system to the installation.

\section{IMPACT EVALUATION}

The system entered full operation during the 2010 winter. The Cray consumes around $500 \mathrm{~kW}$ in normal operation. Of that, around $10 \%$ is used in the power supplies and other parts cooled by air not captured by the heat reuse system. Moreover, an additional $30 \%$ is lost due to the fact that the heat exchangers only lower the temperature to around $21^{\circ} \mathrm{C}$ and the CRAC units are used for the additional $5{ }^{\circ} \mathrm{C}$ required to reach the under-floor temperature of $16{ }^{\circ} \mathrm{C}$. The system can be effectively used around $50 \%$ of the year thanks to the climate in Stockholm. By 2012, the system was running stably, after

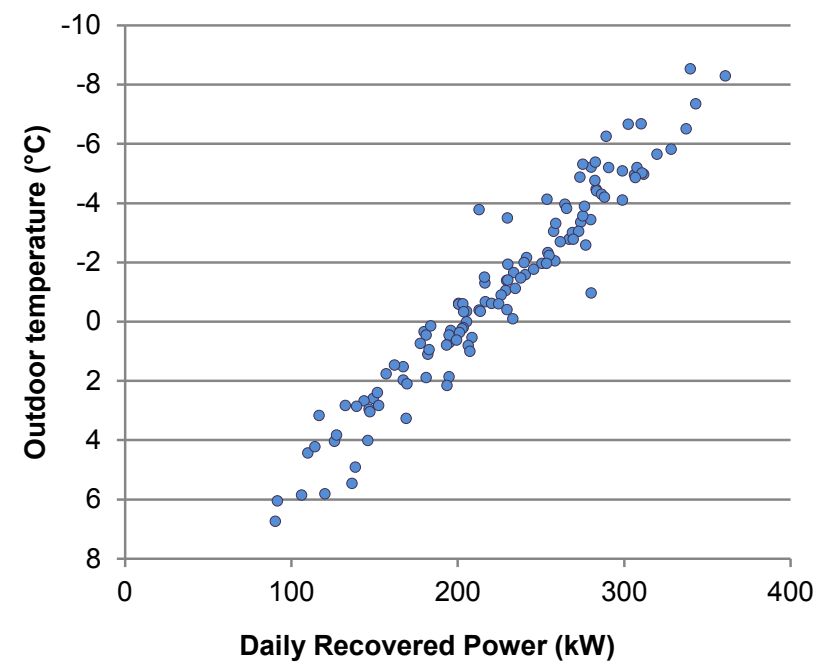

Fig. 9. Outdoor temperature vs. daily recovered power. Note the negative axis for temperature. There is a strong positive correlation of 0.97 between recovered power and outdoor chill. several optimizations and calibrations.

We have collected and analyzed the data on the recovered power used for heating the Chemistry building in the 2012-13 Stockholm winter. The data is from the main period of stable use over which we recovered and recycled heat into the Chemistry building. The period of 158 days runs from the 25th of October, 2012 to the 29th of March, 2013. The campus facilities managers, Akademiska Hus, provided the data. We compare the recovered power data to the daily outdoor temperature in Stockholm during the same period (see figures 8 and 9) [19].

Like most supercomputers, Lindgren's work schedule is relatively evenly distributed throughout the day and week. In fact, most of the time, it hovers at around $90 \%$. Therefore, the differences in recovered power mainly depend on the demand for heating from the Chemistry building. More heat can be recovered the colder the days are. We compute the correlation between temperature and recovered power to be 0.97 (see figure 9).

From the recovered power we have calculated the recovered energy. Over the analyzed period, the Chemistry building reused, on average, 5.4 MWh of heat from Lindgren per day. This aggregates to $850 \mathrm{MWh}$ during the whole period. In addition to the heat from Lindgren, the Chemistry building recycles hot air through the ventilation system, which further reduces the need of district heating. The recycled air has to be taken into account when calculating Lindgren's contribution to savings in district heating. According to Akademiska Hus, about $50 \%$ of the recovered heat from Lindgren is replacing district heating. The other part is replacing heat that could have been provided by recycling air. Based on our calculations on recovered energy, that makes the average savings in district heating about $2.7 \mathrm{MWh}$ per day and the total savings over the period $430 \mathrm{MWh}$.

Fortum, the district heating provider in Stockholm, estimates the average emissions of district heating to be 114.3 $\mathrm{g} / \mathrm{kWh}$ for 2013 [20]. From that, we estimate that the recovered heat from Lindgren reduced the carbon emissions by approximately 300 kilograms per day and nearly 50 tons in 
total over the 158-day winter period in 2012-13. To provide perspective, the yearly average $\mathrm{CO} 2$ emission per capita in Sweden in 2010 was 5.6 metric tons [21].

The reuse of heat from Lindgren also resulted in substantial economic savings. With a cost of $0.07 € / \mathrm{kWh}$ for district heating, KTH saved approximately $30,000 €$ over the analyzed period. In addition, costs for cooling Lindgren were reduced with approximately $40,000 €$, since $\mathrm{KTH}$ is charged 0.05 $€ / \mathrm{kWh}$ for the disposal of the heat through the district cooling system.

\section{DISCUSSION}

We discuss three main points. First, the nature of digital computing and its inseparability from heat production. Second, the mentality of free digital waste and how it is polluting data centers with unnecessary bits. And third, the lessons learned in the design of Lindgren's heat recovery system that go beyond the related work lessons.

\section{A. Computing and Heat}

Computing inexorably produces heat as it pushes electrons through circuits with electrical impedance. Landauer's Principle states that there exists a lower theoretical limit of energy consumption of a computation called the Landauer Limit [22]. The principle predicts that there is a minimum possible amount of energy required to change one bit of information:

\section{$k T \ln 2$,}

where $k$ is the Boltzmann constant $(1.38 \times 10-23 \mathrm{~J} / \mathrm{K}), T$ is the working temperature of the circuit in kelvins, and $\ln 2$ is the natural logarithm of 2 . Theoretically, computers operating at room temperature could flip one billion bits per second with 2.85 trillionths of a watt of power [23]. Consider Tianhe-2 with 33.8 peta-FLOPs consuming $17.8 \mathrm{MW}$. The 33.8 peta-FLOPs conservatively translate to roughly to $4.12 \times 10^{20}$ bit operations per second. Thus, the theoretical efficiency of Tianhe- 2 is roughly $0.0000066 \%$. Evidently, we have a long way to go in computing systems that approximate theoretical efficiency. Nevertheless, the point here is that no matter how efficient they get, computing will always produce heat. As such, large computing centers which produce usable-grade heat should always be regarded as plants that harvest two intertwined products with commercial value: computation and heat.

\section{B. Cloud Data Pollution}

Our activities in the digital world are creating mountains of data through our many social media posts, photographs, locations, and file sharing. This data is in many cases extremely redundant, trivial, and unnecessary. Arguably, we are polluting the digital world with junk bits, yet current interaction design has done little to address the notion that these bits need to live somewhere and that keeping them alive has real-world energy and pollution costs. As stated in the introduction, data centers are consuming billions of watts of electricity, yet it is unclear how much of this is wasteful from a data-centric perspective beyond the thermodynamics. We have several open research questions. First, what mental models do people who create and share digital content have of where their "cloud" data resides and how it is maintained at what environmental cost? Second, how can we design interactivity to shed light on the costs associate with data cloud storage? Third, how can these designs raise awareness and modify behavior towards a more sustainable data storage culture? Finally, how do we measure the mass impact of these proposed changes towards reducing digital waste contributing to the heat production in data centers? These questions apply to data centers. What can be done to improve the culture of sustainable consumption of supercomputing centers?

\section{Novel design and operation lessons}

Previous work predicted or demonstrated many principles of operation for heat recovery systems in supercomputer centers. Namely, Brunschwiler et al. predicted that two important components in moving towards zero-emission supercomputer centers are more efficient cooling and reuse of the centers' waste heat [9]. Furthermore, Ebrahimi et al. observed that reusing waste heat requires minimum supply temperatures to the district heating lines, the benefits of heat reuse for air and water heating include a reduction on operating costs and potential income from selling the heat. The challenges of this type of deployments include greater system complexity and possible maintenance and security issues [4]. Finally, they claim district heating may not be available, depending on the location of the center. In this paper, we have demonstrated all of these principles and advantages. Yet, these experiences are only a subset of the lessons we learned.

First, and most importantly, we demonstrated an original design for using district cooling lines in reverse direction to heat buildings normally upstream from the computer center. We deployed minimal installation requirements, using existing infrastructure to divert the normal flow of cool water and replace it with low-grade recovered heat.

Second, we demonstrated special opportunities in academic and industrial campuses. Our service of the Chemistry building is an excellent example of this extra advantage. Given the extraordinary need for aeration to divert hazardous fumes, the Chemistry building requires constant air heating. This necessity not only maximizes the efficiency of the heat recycling system, it also secures the cooling capacity of the returning water to the computer center. This reduces the computer center needs for purchasing cooling water from the district distributor, thus lowering cost and improving sustainability.

Third, an unforeseen advantage of recycling heat is the creation of a backup cooling system. During our operation of the system, district cooling failed a number of times. In all these instances the heat recovery system provided the necessary cooling capacity to maintain Lindgren operating without interruption. The original back up system through tap water became, effectively, a second back up system that we never needed to deploy. Thus, we reduced operation costs and increased sustainability and reliability.

\section{CONCLUSION AND FUTURE WORK}

We have presented the design, implementation, and evaluation of an original and unique supercomputing heat recovery system in uninterrupted operation for more than four years. The system collects heat from a large-scale 
supercomputer through air cooling and transfers the heat through a large industrial heat exchanger to water pipes that feed into the district cooling lines running in reverse flow during the winter. The computer center heats the Chemistry building that is normally upstream, but downstream when in reversed flow. We have measured the environmental impact of the system during a 158-day winter period and calculated a carbon emission reduction of 50 tons over this time. We measured the energy consumption reduction costs over the same period to be approximately $70,000 €$. Finally, we demonstrated that the heat recovery system served, in practice, as an additional back up layer providing extra protection from overheating to the supercomputing center. Yet, the most important lesson from this paper is that while the speed and power requirements of supercomputers will probably never cease to increase, there are concrete measures designers and operators can take to reduce, reuse, and recycle energy in these types of systems. These measures save money, reduce carbon footprint, and make the system more reliable. As stated in the green 500, supercomputers should be part of a sustainable environmental solution through powerful weather simulations, not part of the problem.

As supercomputers have a very short life time, we have begun the process of procurement for the next system. While the heat transfer systems deployed for Lindgren will most probably be removed from the center, the external heat exchangers to the district cooling system will remain. We aim at maturing our sustainable designs through the deployment of new water-cooled supercomputers like the Cray XC30 which will raise the transfer efficiency of the heat recycling system.

\section{ACKNOWLEDGMENT}

Research funded by the Swedish e-Science Research Council SeRC and the European PRACE First Phase Implementation Project.

\section{REFERENCES}

[1] J. Glanz "Power, Pollution and the internet," in New York Times; Sept 23, 2012, Sect. A:1.

[2] http://data.worldbank.org/topic/energy-and-mining, accessed March 10, 2014.

[3] http://www.top500.org/statistics/, accessed March 10, 2014.

[4] K. Ebrahimi, G. F. Jones, and A. S. Fleischer, "A review of data center cooling technology, operating conditions and the corresponding low-grade waste heat recovery opportunities," in Renewable and Sustainable Energy Reviews 31, 2014: 622-638.

[5] B. Subramaniam and W. Feng, "The Green Index: A Metric for Evaluating System-Wide Energy Efficiency in HPC Systems," in Proceedings of the 8th IEEE Workshop on High-Performance, Power-Aware Computing (HPPAC), Shanghai, China, May 2012.

[6] http://green500.org/, accessed March 10, 2014.

[7] B. Subramaniam, et al. "Trends in energy-efficient computing: A perspective from the Green500," in Green Computing Conference (IGCC), 2013 International. IEEE, 2013.
[8] S. Sharma, C-H. Hsu, and W. Feng, "Making a case for a green500 list," in Parallel and Distributed Processing Symposium, 2006. IPDPS 2006. 20th International. IEEE, 2006.

[9] T. Brunschwiler, B. Smith, E. Ruetsche, \& B. Michel, "Toward zero-emission data centers through direct reuse of thermal energy, "IBM Journal of Research and Development, 53(3), 2009: 11-1.

[10] M. Iyengar, M. David, P. Parida, V. Kamath, B. Kochuparambil, Graybill, D. "Server liquid cooling with chiller-less data center design to enable energy savings," in Proceedings of the $28^{\text {th }}$ IEEE SEMI-THERM symposium. San Jose (CA, USA); 18-22 March, 2012: 212-23.

[11] H. E. Khalifa, and D. W. Demetriou, "Energy optimization of air-cooled data centers," in Journal of Thermal Science and Engineering Applications 2.4, 2010: 041005.

[12] J. Liu, et al., "The data furnace: heating up with cloud computing," in The 3rd USENIX Workshop on Hot Topics in Cloud Computing (HotCloud'11), 2011.

[13] J. Vela, "Helsinki data centre to heat homes," in The Guardian, 2010, July 20.

http://www.guardian.co.uk/environment/2010/jul/20/helsinkidata-centre-heat-homes Accessed on March 10, 2014.

[14] J. Nicolai, "Swedish data center saves \$1 million a year using seawater for cooling," in PCWorld, May 16, 2013.

http://www.pcworld.com/article/2038934/swedish-data-centersaves-1-million-a-year-using-seawater-for-cooling.html, accessed on March 10, 2014.

[15] B. Gardiner, "Tech Incubators Focus on Keeping Europe Green", The New York Times, March 9, 2014. http://www.nytimes.com/2014/03/10/technology/techincubators-focus-on-keeping-europe-green.html

[16] G. Svensson and J. Söderberg, "A Heat Re-Use System for the Cray XE6 and Future Systems at PDC, KTH," in Cray User Group Final Proceedings: Greengineering the Future. Cray User Group, 2012.

[17] Summary of energy statistics for dwellings and non-residential premises 2010, ES 2011:11, ISSN 1654-7543, Swedish Energy Agency, available at http://www.energimyndigheten.se.

[18] Hot Aisle vs. Cold Aisle Containment for Data Centers, White Paper 135, John Niemann, Kevin Brown, Victor Avelar, APC by Schneider Electric, http://www.findwhitepapers.com/content14387.

[19] The Stockholm - Uppsala County Air Quality Management Association, http://slb.nu/lvf/, accessed on March 10, 2014.

[20] Fortum, Värme och miljön, Stockholm, 2013. http://www.fortum.com/countries/se/SiteCollectionDocuments/ miljoredovisning-fortum-varme-2013.pdf

[21] http://data.worldbank.org/indicator/EN.ATM.CO2E.PC accessed May 31, 2014.

[22] R. Landauer, "Irreversibility and heat generation in the computing process." IBM journal of research and development 5.3 (1961): 183-191

[23] http://tikalon.com/blog/blog.php?article=2011/Landauer accessed May 31, 2014. 